J. Austral. Math. Soc. 19 (Series A) 1975, 343-357.

\title{
2-GROUPS OF ALMOST MAXIMAL CLASS
}

\author{
RODNEY JAMES
}

(Received 21 January 1973, revised 12 October 1973)

Communicated by G. E. Wall

\section{Introduction}

Let $G$ be a group of order $2^{n}$ and $x, y \in G$. We define the commutator $[x, y]$ as $x^{-1} y^{-1} x y$. Similarly, if $X, Y$ are subsets of $G$, then $[X, Y]$ denotes the subgroup generated by all commutators of the form $[x, y]$ where $x \in X, y \in Y$. Using this, we may define the lower central series of $G$ inductively by

$$
\gamma_{2}(G)=[G, G], \gamma_{i+1}(G)=\left[\gamma_{i}(G), G\right], \quad(i \geqq 2) .
$$

The following results are well known.

THEOREM 1.1. Let $G$ be a group of order $2^{n}$. Then

(a) there exists a positive integer $c$ (known as the class of $G$ ) such that $\gamma_{i}(G)=1$ (the identity group) if and only if $i>c$.

(b) $c<n$ (and so we can speak of a group of maximal class in the case $c=n-1)$.

(c) If $n>3$, there are precisely three groups of maximal class (to within isomorphism). *

The purpose of this paper is to find all the groups of "almost maximal" class, i.e. when the class is $n-2$. The main result is

THEOREM 1.2. The number of groups of order $2^{n}$ and class $n-2$ for $n>4$ is:*

(a) 15 when $n=5$

(b) 22 when $n=6$

(c) 26 when $\mathrm{n}=7$

(d) $24+4(n, 2)$ when $n>7$, where $(n, 2)=1$ for $n$ odd and $(n, 2)=2$ for $n$ even.

Incidentally, theorem 4.3 also lists the groups in the class $\operatorname{ECF}(m, n, 2)$ of groups described by Blackburn (1958).

* Throughout this paper, "number of groups" is to be understood to mean "number of isomorphism classes of groups". 


\section{Preliminaries}

Most of the concepts and results of this paper may be found in the paper by Blackburn (1958) and the interested reader is referred to that paper. All groups in this paper will be assumed to be finite 2-groups (groups of order $2^{n}$ for some integer $n$ ).

The notation $C_{r}$ will be used for the abstract cyclic group of order $r$ and if $G, H$ are two groups $G \times H$ will denote the direct product of $G$ and $H$. In particular, an elementary abelian 2-group is one of the form $C_{2} \times C_{2} \times \cdots \times C_{2}$. If $G$ is a group, we will write $|G|$ for the order $G, H \leqq G$ to denote that $H$ is a subgroup of $G, H<G$ to denote that $H$ is a proper subgroup of $G(H \neq G)$ and $H \triangleleft G$ to denote that $H$ is a proper normal subgroup of $G$. Also if $x, y, x_{1}, x_{2}, \cdots x_{n}$ $\in G, X \subseteq G, H \leqq G$ and $N \triangleleft G$, then

$$
\begin{gathered}
x^{y}=y^{-1} x y=x[x, y] \\
{\left[x_{1}, x_{2}, \cdots, x_{n}\right]=\left[\left[x_{1}, x_{2}, \cdots, x_{n-1}\right], x_{n}\right] \text { for } n>2}
\end{gathered}
$$

$\left\langle x_{1}, x_{2}, \cdots, x_{n}\right\rangle$ (or $\langle X\rangle$ ) is the subgroup generated by $x_{1}, x_{2}, \cdots, x_{n}$ (or $X$ )

$$
\begin{aligned}
& \mathscr{C}(x)=\{z \in G \mid x z=z x\} \text { is the centralizer of } x \\
& \mathscr{C}(X)=\{z \in G \mid x z=z x \text { for all } x \in X\} \\
& G-X=\{g \in G \mid g \notin X\} \\
& |G: H|=\text { number of right cosets of } H \text { in } G
\end{aligned}
$$

$G / N$ is the group whose elements are the cosets of $N$

Finally, $\Phi(G)$ denotes the Frattini subgroup of $G$ (the intersection of the maximal subgroups of $G$ ) and $Z_{i}(G)$ denotes the $i$-th term of the upper central series defined inductively by

$$
Z_{1}(G)=Z(G)=\mathscr{C}(G), Z_{i+1}(G) / Z_{j}(G)=Z\left(G / Z_{i}(G)\right) \quad i \geqq 1
$$

The following results will be used throughout this paper.

THEOREM 2.1. Let $G$ be a group generated by a set $X$ of elements. If $\gamma_{i}(G)=\left\langle Y, \gamma_{i+1}(G)\right\rangle$, then $\gamma_{i+1}(G)=\left\langle[X, Y], \gamma_{i+2}(G)\right\rangle$ for all $i \geqq 1$ (where $\gamma_{1}(G)$ is interpreted to mean $G$ for this theorem only).

Proof. See Hall (1933), Theorem 2.81.

THEOREM 2.2. Let $G$ be a group and $x, y, z \in G$. Then

(a) $[x y, z]=[x, z]^{y}[y, z],[x, y z]=[x, z][x, y]^{z}$

(b) $\left[x^{-1}, y\right]=[y, x]^{x^{-1}},\left[x, y^{-1}\right]=[y, x]^{y^{-1}}$

(c) $\left[x, y^{2}\right]=[x, y]^{2}[x, y, y],\left[x^{2}, y\right]=[x, y]^{2}[x, y, x][[x, y, x],[x, y]]$

(d) $(x y)^{2}=x^{2} y^{2}[y, x][y, x, y]$ 
THEOREM 2.3. Let $G$ be a group and $x, y, z \in G$. Then

$$
[x, y, z]=[z, x]^{w}[z, y]^{x w}[x, z]^{y}[y, z] \text { where } w=[x, y]
$$

ProOF. $[w, z]=\left[x^{-1} x w, z\right]=\left[x^{-1}, z\right]^{x w}\left[y^{-1} x y, z\right]$

$$
\begin{aligned}
& =[z, x]^{w}\left[y^{-1}, z\right]^{x y}[x, z]^{y}[y, z] \\
& =[z, x]^{w}[z, y]^{x w}[x, z]^{y}[y, z] \text { by theorem 2.2.(b). }
\end{aligned}
$$

Corollary. If $\gamma_{2}(G)$ is abelian, $[x, y, z]=[z, y, x][x, z, y]$

THEOREM 2.4. Let $G$ be a group of order $2^{n}$ and class $n-1$. Then $G$ has a cyclic subgroup $\left\langle s_{1}\right\rangle$ of index 2 such that $\gamma_{i}(G)=\left\langle s_{1}^{2^{i-1}}\right\rangle$ for all $\left.i\right\rangle 1$. If $\left.n\right\rangle 3$, there are three choices for $G$ with generators $s, s_{1}$ and the defining relations (where $u=s_{1}^{2 n-2}$ ):

(i) $u^{2}=s_{1}^{2}\left[s_{1}, s\right]=s^{2}=1$,

(ii) $u^{2}=s_{1}^{2}\left[s_{1}, s\right]=1, s^{2}=u$,

denoted $D_{n}$

(iii) $u^{2}=s^{2}=1, s_{1}^{2}\left[s_{1}, s\right]=u$, denoted $Q_{n}$ denoted $S_{n}$

If $n=3$, (iii) defines an abelian group and so there are only two choices for $G$ in this case.

Proof. See Hall and Senior (1964), page 9.

For the next theorem, we need two more concepts. If $G$ and $H$ are two groups whose centres are isomorphic, we may construct a group (called a central product of $G$ and $H$ ) consisting of all formal products $g h$ with $g \in G, h \in H$ where elements in $Z(G)$ and $Z(H)$ which correspond under the above isomorphism are regarded as the same and where $(g h)\left(g^{\prime} h^{\prime}\right)=\left(g g^{\prime}\right)\left(h h^{\prime}\right)$ for all $g, g^{\prime} \in G$ and $h, h^{\prime} \in H$. Also a 2-group $G$ of class 2 with $G / \gamma_{2}(G)$ elementary abelian and $|Z(G)|=\left|y_{2}(G)\right|=2$ is called an extra-special 2-group.

THEOREM 2.5. (a) An extra-special 2-group is isomorphic to the central product of several copies of $D_{3}$ and at most one copy of $Q_{3}$.

(b) A 2-group $G$ of class $c>2$ with $|Z(G)|=2$ is the central product of an extra special 2-group and a group $L$ with $|Z(L)|=2$ and $Z_{2}(L)$ abelian.

Proof. See Hall and Senior (1964), Theorems 4.1 and 4.2. Their proof of (b) for $c=3$ is easily adapted to the general case.

\section{CF $(m, n, 2)$}

A useful set of groups of order $2^{n}$ is the set $\operatorname{CF}(m, n, 2)$ of groups $G$ whose class is $m-1$ and

$$
\left|\gamma_{i}(G): \gamma_{i+1}(G)\right|=2 \quad i \geqq 2
$$


If $G \in \mathrm{CF}(m, n, 2)$ and $m>3$ we define a subgroup $\gamma_{1}(G)$ of $G$ by the property that $\gamma_{1}(G) / \gamma_{4}(G)$ is the centraliser in $G / \gamma_{4}(G)$ of $\gamma_{2}(G) / \gamma_{4}(G)$.

Theorem 3.1. (Blackburn (1958)). Let $G \in \mathrm{CF}(m, n, 2)$ and $m>3$. Then (a) $G>\gamma_{1}(G)>Z_{m-2}(G) \geqq \gamma_{2}(G)$ and $\left|G: \gamma_{1}(G)\right|=\left|\gamma_{i}(G): Z_{m-2}(G)\right|=2$,

(b) If $r$ is the smallest positive integer such that there exists $s \in G-\gamma_{1}(G)$ with $s^{2 r} \in \gamma_{2}(G)$, then $\gamma_{2}(G)$ can be generated by less than $2^{r}$ elements.

If $s$ is an element of $G$ with $s \notin \gamma_{1}(G)$ and $s \notin \mathscr{C}\left(\gamma_{m-2}(G)\right)$, and $s_{1} \in \gamma_{1}(G)-Z_{m-2}(G)$, we may define elements inductively by

$$
s_{i+1}=\left[s_{i}, s\right] \quad i=1,2, \cdots
$$

THEOREM 3.2. Let $G \in \mathrm{CF}(m, n, 2)$ with $m \geqq 5$ and $s, s_{i}$ as above. Then $\mathscr{C}(s) \cap \gamma_{2}(G) \leqq \gamma_{4}(G)$ and $\gamma_{i}(G)=\left\langle s_{i}, \gamma_{i+1}(G)\right\rangle, i=2,3,4$.

Proof. By Theorem 2.1, $\gamma_{2}(G)=\left\langle s_{2}, \gamma_{3}((G)\rangle\right.$, and, since $\left[\gamma_{1}(G), \gamma_{2}(G)\right]$ $\leqq \gamma_{4}(G)$, we get $\gamma_{3}(G)=\left\langle s_{3}, \gamma_{4}(G)\right\rangle$, and so $\gamma_{4}(G)=\left\langle\left[s_{3}, \gamma_{1}(G)\right]\right.$, $\left.s_{4},\left[s_{3}, \gamma_{1}(G)\right], \gamma_{5}(G)\right\rangle$. However, if $v \in \gamma_{1}(G)$, then

$$
\begin{aligned}
{\left[s_{3}, v\right]=\left[s_{2}, s, v\right] } & \equiv\left[s_{2}, v, s\right]\left[v, s, s_{2}\right]\left(\bmod \gamma_{5}(G)\right) \\
& \equiv\left[v, s, s_{2}\right]\left(\bmod \gamma_{5}(G)\right) \text { since }\left[s_{2}, v\right] \in \gamma_{4}(G)
\end{aligned}
$$

But $[v, s] \in\left\langle s_{2}, \gamma_{3}(G)\right\rangle$ and so

$$
\left[v, s, s_{2}\right] \in\left[\gamma_{3}(G), \gamma_{2}(G)\right] \leqq \gamma_{5}(G) .
$$

Thus $\left[s_{3}, v\right] \in \gamma_{5}(G)$ and so $\gamma_{4}(G)=\left\langle s_{4}, \gamma_{5}(G)\right\rangle$. If $m \geqq 5, s_{4} \notin \gamma_{5}(G)$ and so $s_{3} \notin \mathscr{C}(s)$, giving the results.

The above result may be improved if we assume a condition of commutativity on $\gamma_{1}(G)$ :

TheOREM 3.3. (Blackburn (1958)). Let $G \in C F(m, n, 2)$ with $m>3$ and suppose $\left[\gamma_{1}(G), \gamma_{i}(G)\right] \leqq \gamma_{i+2}(G)$ for $i=1,2, \cdots m-3$. If $s, s_{i}$ are defined as above then

(a) $\gamma_{i}(G)=\left\langle s_{i}, \gamma_{i+1}(G)\right\rangle, i=2,3, \cdots m-1$

(b) $\mathscr{C}(s) \cap \gamma_{2}(G)=\gamma_{m-1}(G)$

(c) There exists a set $T$ of elements of $\mathscr{C}(s)$ such that $Z_{m-2}(G)=\left\langle T, \gamma_{2}(G)\right\rangle$ and $[t, u] \in \gamma_{m-1}(G)$ for all $t, u \in T$.

Corollary 1. $|\mathscr{C}(s)|=2^{n-m+2}$.

COROLlary 2. The conjugacy class containing $s$ is $s \gamma_{2}(G)$.

Since we will be assuming that $G / \gamma_{2}(G)$ has exponent 2 or 4 , we shall consider that case now.

THEOREM 3.4. Let $G \in C F(m, n, 2)$ with $m>3$ and suppose $G / \gamma_{2}(G)$ has exponent $2^{r}$ with $r \leqq 2$. If $s, s_{i}$ are defined as above, then 
(a) $\left[\gamma_{1}(G), \gamma_{i}(G)\right] \leqq \gamma_{i+2}(G)$ for $i$ odd

(b) $\gamma_{i}(G)=\left\langle s_{i}, \gamma_{i+1}(G)\right\rangle, i=2,3, \cdots m-1$

(c) $\mathscr{C}(s) \cap \gamma_{2}(G)=\gamma_{m-1}(G)$

(d) If $\gamma_{2}(G)$ may be generated by 2 elements, $G$ satisfies the conditions of theorem 3.3.

Proof. By theorem 3.1, $\gamma_{2}(G)$ has at most three generators. Since $\Phi\left(\gamma_{2}(G)\right) \triangleleft G, \Phi\left(\gamma_{2}(G)\right)=\gamma_{2+d}(G)$ where $d$ is the number of generators of $\gamma_{2}(G)$.

If $d \leqq 2$, then by theorem 2 of Blackburn (1957), $\gamma_{3}(G) \leqq Z\left(\gamma_{2}(G)\right)$ and so $\gamma_{2}(G)$ is abelian. By the corollary to theorem 2.10 of Blackburn (1958), $G$ then satisfies the conditions of theorem 3.3, proving all parts of this theorem for this case.

If $d=3$, we have $\Phi\left(\gamma_{2}(G)\right)=\gamma_{5}(G)$ and so $s_{2}^{2}, s_{3}^{2}, s_{4}^{2} \in \gamma_{5}(G)$. Writing $\bar{G}$ for $G / \gamma_{6}(G)$, theorem 3.2 tells us that $\bar{G}$ satisfies the condition for theorem 3.3 and so (b) is true for $i \leqq 5$. Also since $s_{2}^{2}, s_{3}^{2} \in \gamma_{5}(G)$ and $s^{4} \in \mathscr{C}(s) \cap \gamma_{2}(G) \leqq \gamma_{5}(G)$, we get (working modulo $\gamma_{6}(G)$ )

$$
\begin{aligned}
s_{4}^{2} & \equiv s_{5}^{2} \equiv 1, \\
s_{3}^{2}\left[s_{3}, s_{2}\right] & \equiv\left[s_{2}^{2}, s\right] \equiv 1, \\
1 \equiv\left[s_{1}, s^{4}\right] & \equiv\left[s_{1}, s^{2}\right]^{2}\left[s_{1}, s^{2}, s^{2}\right] \\
& \equiv\left(s_{2}^{2} s_{3}\right)^{2}\left[s_{3}, s^{2}\right] \equiv s_{3}^{2} s_{5}
\end{aligned}
$$

and, for $v \in \gamma_{1}(G),\left[s_{4}, v\right]=\left[s_{3}, s, v\right]$

$$
=\left[v, s_{3}\right]^{s_{4}}[v, s]^{s_{3} s_{4}}\left[s_{3}, v\right]^{s}[s, v] \equiv\left[v, s, s_{3}\right]
$$

$$
\text { i.e. } s_{3}^{2} \equiv\left[s_{3}, s_{2}\right] \equiv\left[s_{4}, s_{1}\right] \equiv s_{5}\left(\bmod \gamma_{6}(G)\right)
$$

and

$$
\left[s_{4}, v\right] \equiv \begin{cases}s_{5} & \text { if }[v, s] \notin \gamma_{3}(G) \\ 1 & \text { if }[v, s] \in \gamma_{3}(G)\end{cases}
$$

We now prove (by induction) the following:

(i) $\left[s_{2 j}, s_{1}\right] \equiv s_{2 j+1}\left(\bmod \gamma_{2 j+2}(G)\right),\left[s_{2 j-1}, s_{1}\right] \in \gamma_{2 j+1}(G) \quad j \geqq 2$

(ii) $s_{i} \equiv s_{i-2}^{2}\left(\bmod \gamma_{i+1}(G)\right) \quad i \geqq 5$.

Since these results have been proved for $j=2$ and $i=5$, we will assume them for $2 \leqq j^{\prime} \leqq j, 5 \leqq i^{\prime} \leqq i$. Then $s_{i+1}=\left[s_{i}, s\right] \equiv\left[s_{i-2}^{2}, s\right] \equiv s_{\imath-1}^{2}\left(\bmod \gamma_{i+2}(G)\right)$, proving (ii). Also

$$
\left[s_{2(j+1)}, s_{1}\right] \equiv\left[s_{2 j}^{2}, s_{1}\right] \equiv\left[s_{2 j}, s_{1}\right]^{2} \equiv s_{2 j+1}^{2} \equiv s_{2 j+3}\left(\bmod \gamma_{2 j+4}(G)\right)
$$

and similarly $\left[s_{2 j+1}, s_{1}\right] \in \gamma_{2 j+3}(G)$, proving (i).

Using these results, we may show in a similar way that, for $v \in \gamma_{1}(G)$, $\left[s_{2 j+1}, v\right] \in \gamma_{2 j+3}(G)$ yielding (a), and $\left[v, s_{2 j}\right] \equiv s_{2 j+1}$ or $1\left(\bmod \gamma_{2 j+2}\right)$. Thus, if $\gamma_{i}(G)=\left\langle s_{i}, \gamma_{i+1}(G)\right\rangle$, then 
proving (b).

$$
\begin{aligned}
\gamma_{i+1}(G) & =\left\langle s_{i+1},\left[s_{i}, \gamma_{1}(G)\right], \gamma_{i+2}(G)\right\rangle \\
& =\left\langle s_{i+1}, \gamma_{i+2}(G)\right\rangle
\end{aligned}
$$

Finally, (c) follows from (b) which tells us that $\left[s_{i}, s\right] \neq 1$ and so $s_{i} \notin \mathscr{C}(s)$ for $i=2,3, \cdots m-2$.

COROLlaRY. If $s^{2} \in \gamma_{2}(G)$, then $\left\langle s, \gamma_{2}(G)\right\rangle$ is a group of maximal class for $m>3$ and $s_{i}^{2} s_{1+1}=1$ for $i=2,3, \cdots m-2$.

PROOF. $\left\langle s, \gamma_{2}(G)\right\rangle$ has order $2^{m-1}$ and class $m-2$. Clearly $s^{2} \in \mathscr{C}(s) \cap \gamma_{2}(G)$ $=\gamma_{m-1}(G) \leqq Z(G)$ and so $1=\left[s_{j}, s^{2}\right]=\left[s_{j}, s\right]^{2}\left[s_{j}, s, s\right]=s_{j+1}^{2} s_{j+2}$ for $j=1,2$, $\cdots m-2$.

\section{ECF $(m, n, 2)$}

Following Blackburn, we say that a group $G$ belongs to the class $\operatorname{ECF}(m, n, 2)$ if $G \in \mathrm{CF}(m, n, 2)$ and $G / \gamma_{2}(G)$ is elementary abelian. In this paragraph we will determine all the groups of $\operatorname{ECF}(m, n, 2)$ for any values of $m, n$ with $m>2$. In particular, it is clear that $\operatorname{ECF}(n, n, 2)$ consists of the groups of order $2^{n}$ and maximal class and that the cyclic subgroup of index 2 in theorem 2.4 is the subgroup $\gamma_{1}(G)$.

Since $\operatorname{ECF}(m, n, 2) \subset \operatorname{CF}(m, n, 2)$, we may use the results of the previous section. Thus if $G \in \operatorname{ECF}(m, n, 2)$ then $\gamma_{2}(G)$ is cyclic by Theorem $3.1(\mathrm{~b})$ and so (by theorem 3.4(d)) there exist elements $s \in G-\gamma_{1}(G), s_{1} \in \gamma_{1}(G)-Z_{m-2}(G)$ and a set $T \subset \mathscr{C}(s)$ such that $G=\left\langle s, \gamma_{1}(G)\right\rangle, \gamma_{1}(G)=\left\langle s_{1}, Z_{m-2}(G)\right\rangle, Z_{m-2}(G)=$ $\left\langle T, \gamma_{2}(G)\right\rangle$ and $\gamma_{i}(G)=\left\langle s_{i}, \gamma_{i+1}(G)\right\rangle$ where $s_{i}=\left[s_{i-1}, s\right]$ for $i=2,3, \cdots m-1$. Also, if we write $G_{1}$ for $\left\langle s, s_{1}\right\rangle$ then it is clear that $\gamma_{i}\left(G_{1}\right)=\gamma_{i}(G)$ for $i=2,3, \cdots m-1$, and $G_{1}$ is a group of order $2^{m}$ and class $m-1$. By theorem 2.4, we can see that

$$
s_{i}^{2} s_{i+1}=\left[s_{i}, s_{j}\right]=1 \quad \text { for all } i \geqq 2, j \geqq 1
$$

and that we may assume

$$
s^{2}=s_{m-1}^{\alpha}, \quad s_{1}^{2} s_{2}=s_{m-1}^{\beta}
$$

where $\alpha, \beta=0$ or 1 and $\alpha, \beta$ are not both equal to 1 .

LEMMA. If $m>3, Z_{2}(G)=\left\langle T, \gamma_{m-2}(G)\right\rangle$ and $t^{2} \in \gamma_{m-1}(G)$ for all $t \in T$.

Proof. Let $t \in T$. As in the case of $G_{1}$, we may show that $\left\langle s, s_{1} t\right\rangle$ is a group of maximal class and so for $i \geq 2,1=\left[s_{i}, s_{1} t\right]=\left[s_{i}, t\right]\left[s_{i}, s_{1}\right]$. Since $\left[s_{i}, s_{1}\right]=1$ and $T \subset \mathscr{C}(s)$,

$$
\left[T, \gamma_{2}(G)\right]=[T, s]=1
$$

To find $\left[s_{1}, t\right]$, we note that $s_{1}^{2} \in \gamma_{2}(G)$ and so

$$
1=\left[t, s_{1}^{2}\right]=\left[t, s_{1}\right]^{2}\left[t, s_{1}, s_{1}\right]=\left[t, s_{1}\right]^{2} .
$$


Since $\gamma_{2}(G)$ is cyclic, $\gamma_{m-1}(G)$ is the only subgroup of order 2 and so $\left[t, s_{1}\right] \in \gamma_{m-1}(G)$ Using Theorem 3.3(c) and the fact that $[t, s]=1$, we see that $[T, G] \leqq \gamma_{m-1}(G)$ $\leqq Z(G)$, and so $\left\langle T, \gamma_{m-2}(G)\right\rangle \leqq Z_{2}(G)$. The equality follows from the facts that

$$
Z_{2}(G) \leqq Z_{m-2}(G)=\left\langle T, \gamma_{2}(G)\right\rangle \text { and } Z_{2}(G) \cap \gamma_{2}(G) \leqq \gamma_{m-2}(G) .
$$

Finally, since $G / \gamma_{2}(G)$ is elementary Abelian, $t^{2} \in \gamma_{2}(G) \cap \mathscr{C}(s)=\gamma_{m-1}(G)$.

If we write $T=\left\{t_{1}, t_{2}, \cdots t_{n-m}\right\}$, where the $t_{i}$ are independent, and

$$
t_{j}^{2}=s_{m-1}^{\gamma_{j}},\left[s_{1}, t_{j}\right]=s_{m-1}^{\delta_{j}} \quad(j=1,2, \cdots n-m)
$$

then the groups $G$ of $\operatorname{ECF}(m, n, 2)$ will be determined by the equations (1) to (4), the parameters $\alpha, \beta, \gamma_{j}, \delta_{j}$ and the commutators of elements of $T$. The argument proceeds as in paragraph 4 of Blackburn (1958) or the paper by James and Cannon (1969).

THEOREM 4.1. The number of groups $G$ of $\mathrm{ECF}(m, n, 2)$ with $Z_{2}(G)$ abelian and $|Z(G)|=2$ is 2 if $n=m+1>4,3$ if $n=m \neq 3$, and 0 otherwise.

Proof. Since $Z_{2}(G)$ is abelian, we have

$$
\left[t_{i}, t_{j}\right]=\left[s, t_{j}\right]=1 \quad i, j=1,2, \cdots n-m .
$$

Since $|Z(G)|=2$, we must have $\left[s_{1}, t\right]=s_{m-1}$ for all $t \in T$. However, if $\left[s_{1}, t_{1}\right]=\left[s_{1}, t_{2}\right]=s_{m-1}$, then $\left[s_{1}, t_{1} t_{2}\right]=1$ and so $t_{1} t_{2} \in Z(G)$ which is impossible. Thus $n=m$ or $m+1$.

If $n=m$, then $G$ has maximal class and so the result follows. If $n=m+1$ then

$$
G=\left\langle s, s_{1}, t, \gamma_{2}(G)\right\rangle \text { where }\left[s_{1}, t\right]=s_{m-1}, t^{2}=s_{m-1}^{\gamma} .
$$

By replacing $s$ by $s s_{1}^{\gamma+1}$ and $t$ by $t s_{m-2}^{\gamma+1}$, we may suppose $t^{2}=s_{m-1}$. Then, by replacing $s$ by $s t^{x}$, we may suppose $s^{2}=1$. The two choices for $s_{1}^{2} s_{2}$ clearly yield non-isomorphic groups.

If $m=3$, there are no such groups since $Z_{2}(G)=G$ is not abelian.

THEOREM 4.2. The number of groups $G$ of $\operatorname{ECF}(m, n, 2)$ with $|Z(G)|=2$ is 2 if $m=3$ and $n-m$ even or $m \neq 3$ and $n-m$ odd; 3 if $m \neq 3$ and $n-m$ even; and 0 if $m=3$ and $n-m$ odd.

Proof. By Theorem 2.5, $G$ is the central product of groups $G_{1}$ and $C$ where $G_{1}$ is a group of the previous theorem and $C$ is extra special (in the case where $m \neq 3$ ).

(a) If $n-m$ is even, then $G_{1}$ is a group of maximal class and $C=\left\langle t_{1}, \cdots, t_{n-m}\right\rangle$ where $\left[t_{1}, t_{2}\right]=\left[t_{3}, t_{4}\right]=\cdots=\left[t_{n-m-1}, t_{n-m}\right]=s_{m-1},\left[t_{i}, t_{j}\right]=1$ otherwise and

$$
t_{1}^{2}=s_{m-1}^{\gamma}, t_{2}^{2}=t_{3}^{2}=\cdots=t_{n-m}^{2}=s_{m-1} .
$$


By replacing $t_{1}$ by $t_{1} s_{m-2}^{\gamma+1}$ and $s$ by $s t_{2}^{\gamma+1}$, we may suppose $t_{1}^{2}=s_{m-1}$. Since $\mathscr{C}\left(Z_{2}(G)\right)=\left\langle s_{1}\right\rangle$ is characteristic in $G$, we may only replace $s_{1}$ by a power of itself and so the two values for $s_{1}^{2} s_{2}$ yield non-isomorphic groups. If we replace $s$ by $s s_{1}^{\xi} t$ where $\xi$ is a non-negative integer and $t \in Z_{2}(G)-Z(G)$, then we must also replace $t^{\prime}$ by $t^{\prime} s_{m-2}$ (where $t^{\prime}$ is an element of $Z_{2}(G)-Z(G)$ with $\left[t, t^{\prime}\right]=$ $\left.s_{m-1}\right)$ thus changing the value of $t^{\prime 2}$. Hence, if the above values for $t_{i}^{2}\left(t_{i} \in T\right)$ are fixed, the only elements which may replace $s$ are of the form $s s_{1}^{\xi}$, yielding three groups with the relations

$$
\begin{array}{llll}
s_{1}^{2} s_{2} & =s_{m-1} & 1 & 1 \\
s^{2}=1 & s_{m-1} & 1
\end{array}
$$

(b) If $n-m$ is odd, then $G_{1}$ is the other group of theorem 4.1 and $C=\left\langle t_{2}, t_{3}, \cdots t_{n-m}\right\rangle$ where

$$
\left[t_{2}, t_{3}\right]=\left[t_{4}, t_{5}\right]=\cdots=\left[t_{n-m-1}, t_{n-m}\right]=s_{m-1},\left[t_{i}, t_{j}\right]=1
$$

otherwise, and

$$
t_{2}^{2}=s_{m-1}^{\gamma}, t_{1}^{2}=t_{3}^{2}=t_{4}^{2}=\cdots=t_{n-m}^{2}=s_{m-1}
$$

Again, by replacing $t_{2}$ by $t_{2} s_{m-2}^{\gamma+1}$ and $s$ by $s t_{3}^{\gamma+1}$, we may suppose $t_{2}^{2}=s_{m-1}$ and it is again easy to show that the resultant two groups (with $s^{2}=1$ ) are not isomorphic.

If $m=3, G$ is extra-special and the result follows from theorem $2.5(\mathrm{a})$.

THEOREM 4.3. The number of groups in $\operatorname{ECF}(m, n, 2)$ is

$$
\begin{gathered}
{\left[\frac{1}{2}(3 n-5)\right] \text { for } m=3, n \geqq 3} \\
3(n-m+1)+\left[\frac{1}{2}(n-m)\right] \text { for } m>3, n \geqq m
\end{gathered}
$$

where $\left[\frac{1}{2} r\right]=\frac{1}{2} r$ for $r$ even and $\left[\frac{1}{2} r\right]=\frac{1}{2}(r-1)$ for $r$ odd.

Proof. Let $G$ be a group in $\operatorname{ECF}(m, n, 2)$ and use the notation above. Then

$$
Z_{2}(G)=\left\langle t_{1}, t_{2}, \cdots t_{n-m}, \gamma_{m-2}(G)\right\rangle
$$

and, by suitably ordering the $t_{i}$, we may write

$$
\begin{aligned}
Z(G) & =\left\langle t_{l+1}, \cdots, t_{n-m}, s_{m-1}\right\rangle \text { and so } \\
G & =\left\langle G_{2}, t_{l+1}, \cdots t_{n-m}\right\rangle
\end{aligned}
$$

where $G_{2} \in \operatorname{ECF}(m, m+l, 2)$ and $\left|Z\left(G_{2}\right)\right|=2$. Thus $G_{2}$ is one of groups of theorem 4.2 .

If $Z(G)$ has an element of order 4, we may take $t_{l+1}^{2}=s_{m-1}$. By replacing $s$ by $s t_{l+1}^{\alpha}, s_{1}$ by $s_{1} t_{l+1}^{\beta}$ and $t_{j}$ by $t_{j} t_{l+1}^{\gamma_{j}}(j=l+2, \cdots, n-m)$, we may suppose 
and

$$
s^{2}=s_{1}^{2} s_{2}=t_{l+2}^{2}=t_{l+3}^{2}=\cdots=t_{n-m}^{2}=1
$$

$$
t_{1}^{2}=t_{2}^{2}=\cdots=t_{l}^{2}=s_{m-1}
$$

yielding one group for each value of $l=0,1, \cdots n-m-1$ (none for $m=3$ and $l$ odd).

If $Z(G)$ does not have an element of order 4 , it is elementary abelian, and

$$
G=G_{2} \times\left\langle t_{l+1}\right\rangle \times \cdots \times\left\langle t_{n-m}\right\rangle
$$

yielding three groups (two for $m=3$ ) for each even value of $l=0,2, \cdots, n-m$ and two groups (none for $m=3$ ) for each odd value of $l=1,3, \cdots, n-m$.

\section{Groups of order $2^{n}$ and class $n-2$}

In this paragraph, we will use the preceding results to obtain the 2-groups of almost maximal class. The following lemma (the proof of which may be found in Blackburn (1958), page 74) and its corollary will be useful in what follows. class.

LEMMA. If $G$ is a 2-group with $\left|G: \gamma_{2}(G)\right|=4$, then $G$ is a group of maximal

COROLLARY. If $G$ is a group of order $2^{n}$ and class $n-2$, then $G \in \mathrm{CF}(n-1, n, 2)$.

PROOF. Since $\left|\gamma_{i}(G): \gamma_{i+1}(G)\right| \geqq 2$ for $i=2,3, \cdots n-2$, we must have $\left|\gamma_{2}(G)\right|$ $\geqq 2^{n-3}$ and so $\left|G: \gamma_{2}(G)\right| \leqq 8$. By the lemma, $\left|G: \gamma_{2}(G)\right| \neq 4$ and so $\left|\gamma_{2}(G)\right|$ $=2^{n-3}$. Thus $\left|\gamma_{i}(G): \gamma_{i+1}(G)\right|=2$ for each $i$, forcing $G$ to be in CF $(n-1, n, 2)$.

Since the groups of order $2^{n}(n \leqq 4)$ are well known, we will consider only the groups $G$ of order $2^{n}$ and class $n-2$ with $n>4$. In such a group, $\left|\gamma_{1}(G): \gamma_{2}(G)\right|$ $=4$ and $G / \gamma_{2}(G)$ is either of the form $C_{2} \times C_{2} \times C_{2}$ or $C_{4} \times C_{2}$. In the first case, $G \in \operatorname{ECF}(n-1, n, 2)$ and so Theorem 4.3 yields:

THEOREM 5.1. There are precisely 6 groups $G$ of order $2^{n}$ and class $n-2$ $(n>4)$ with $G / \gamma_{2}(G)$ elementary abelian.

In the second case, we have two possibilities depending on whether $\gamma_{1}(G) / \gamma_{2}(G)$ is cyclic or elementary abelian.

THEOREM 5.2. The number of groups $G$ of order $2^{n}$ and class $n-2$ with $\gamma_{1}(G) / \gamma_{2}(G)$ cyclic is 3 if $n=5$ and 6 if $n>5$.

Proof. Since $G \in \mathrm{CF}(n-1, n, 2)$, we may use the results of paragraph 3 and so we may write $G=\left\langle s, s_{1}\right\rangle$ where $\gamma_{1}(G)=\left\langle s_{1}, \gamma_{2}(G)\right\rangle s_{1}^{2} \notin \gamma_{2}(G)$ and $s_{1}^{4} \in \gamma_{2}(G)$. Since $G / \gamma_{2}(G)$ is of the form $C_{4} \times C_{2}$, we may choose $s \notin \gamma_{1}(G)$ such that $s^{2} \in \gamma_{2}(G)$. By theorem 3.4 and its corollary, we have $\gamma_{i}(G)=\left\langle s_{i}, \gamma_{i+1}(G)\right\rangle$ where

$$
s_{i+1}=\left[s_{i}, s\right]=s_{i}^{-2} \text { for } i=2,3, \cdots n-2
$$


To evaluate $\left[s_{1}, s_{i}\right]$, we note that $\left[s_{1}, s_{2}\right] \in \gamma_{4}(G)$. If $r$ is an integer with $r \geqq 4$ such that $\left[s_{1}, s_{2}\right] \in \gamma_{r}(G)-\gamma_{r+1}(G)$, then $\left[s_{1}, s_{2}\right] \equiv s_{r}\left(\bmod \gamma_{r+1}(G)\right)$ and so

$$
\left[s_{1}, s_{3}\right]=\left[s_{1}, s_{2}^{-2}\right] \equiv s_{r}^{-2} \equiv s_{r+1}\left(\bmod \gamma_{r+2}(G)\right) \text {. }
$$

However $\left[s_{1}^{2}, s\right]=\left[s_{1}, s\right]^{2}\left[s_{1}, s, s_{1}\right] \equiv s_{2}^{2} s_{r} \equiv s_{3}\left(\bmod \gamma_{4}(G)\right)$ and so $\left[s_{1}^{4}, s\right]=$ $\left[s_{1}^{2}, s\right]^{2}\left[s_{1}^{2}, s, s_{1}^{2}\right] \equiv s_{3}^{2} \equiv s_{4}\left(\bmod \gamma_{5}(G)\right)$. But $s_{1}^{4} \in \gamma_{2}(G)$ and so $s_{1}^{4} \equiv s_{3}\left(\bmod \gamma_{4}(G)\right)$. Combining these result,

$$
s_{r+1} \equiv\left[s_{1}, s_{3}\right] \equiv\left[s_{1}, s_{1}^{4}\right] \equiv 1\left(\bmod \gamma_{r+2}(G)\right)
$$

which is impossible unless $r \geqq n-2$. Thus

$$
\left[s_{1}, s_{2}\right]=s_{n-2} \text { or } 1, \quad\left[s_{1}, s_{i}\right]=1 \text { for } i>2
$$

Finally, using this result in the above, we get $\left[s_{1}^{4}, s\right]=s_{4}$ and so $s_{1}^{4} s_{3}^{-1} \in \mathscr{C}(s) \cap \gamma_{2}(G)=\gamma_{n-2}(G)$, and so

$$
s^{2}=s_{n-2} \text { or } 1, \quad s_{1}^{4}=s_{3} s_{n-2} \text { or } s_{3}
$$

The equations (1), (2) and (3) are the defining relations for $G$ and it is easy to check (when $n>4$ ) that a group of order $2^{n}$ exists with the given defining relations (with $\left[s_{1}, s_{2}\right]=1$ for $n=5$ ). If $s_{1}^{4} s_{3}^{-1}=s_{n-2}\left[s_{1}, s_{2}\right]$ and $s^{2}=s_{n-2}$ then (by replacing $s$ by $s s_{1}^{2}$ ) we may suppose $s^{2}=1$. Since $\left(s s_{1}^{2}\right)^{2}=s^{2} s_{1}^{4}\left[s_{1}^{2}, s\right]$ $=s^{2} s_{1}^{4} s_{3}^{-1}\left[s_{1}, s_{2}\right]$, we may write the defining relations:

$\begin{array}{lcccccc}{\left[s_{1}, s_{2}\right]} & =1 & 1 & 1 & s_{n-2} & s_{n-2} & s_{n-2} \\ s_{1}^{4} s_{3}^{-1} & =1 & 1 & s_{n-2} & 1 & s_{n-2} & s_{n-2} \\ s^{2} & =1 & s_{n-2} & 1 & 1 & 1 & s_{n-2} \\ \left(s s_{1}^{2}\right)^{2} & =1 & s_{n-2} & s_{n-2} & s_{n-2} & 1 & s_{n-2}\end{array}$

In the first three cases $|Z(G)|=4$ whereas in the last three cases $|Z(G)|=2$ and so none of the first three is isomorphic to any of the last three. In fact, by counting the number of elements of order 2, we can see that each of the above cases yields a distinct group. Thus we obtain three groups for $n=5$ (the groups $\Gamma_{8} c_{1}, \Gamma_{8} c_{2}$ and $\Gamma_{8} e$ of Hall and Senior (1964)) and six groups for $n>5$.

THEOREM 5.3. The number of groups $G$ of order $2^{n}$ and class $n-2$ with $G / \gamma_{2}(G)$ of exponent $4, \gamma_{1}(G) / \gamma_{2}(G)$ elementary abelian and with $\gamma_{2}(G)$ having 1 or 2 generators is 6 for $n=5,8$ for $n=6$ and 10 for $n>6$.

PRoof.Again we use the notation of paragraph 3, noting that $\gamma_{1}(G)=$ $\left\langle s_{1}, s^{2}, \gamma_{2}(G)\right\rangle$. By Theorem 3.4(d), Theorem 3.3 applies and $\gamma_{i}(G)=\left\langle s_{i}, \gamma_{i+1}(G)\right\rangle$ for $i=2,3, \cdots, n-2$ and $s^{4} \in \mathscr{C}(s) \cap \gamma_{2}(G)=\gamma_{n-2}(G) \leqq Z(G)$.

(a) Suppose $\gamma_{2}(G)$ is cyclic and so $\gamma_{2}(G)=\left\langle s_{2}\right\rangle$. If $s_{1}^{2} \in \gamma_{3}(G), s_{2}^{2}=\left[s_{1}^{2}, s\right] \in \gamma_{4}(G)$ contradicting our assumption and so $s_{2} \equiv s_{1}^{2}\left(\bmod \gamma_{3}(G)\right)$. Thus $s^{-1} s_{1} s=s_{1}^{\alpha}$ 
where $\alpha \equiv 3(\bmod 4)$. Since $s^{4} \in Z(G), s_{1}=s^{-4} s_{1} s^{4}=s_{1}^{\alpha^{4}}$ and so $\alpha^{4} \equiv 1\left(\bmod 2^{n-2}\right)$. Solving these congruences, we get $\alpha \equiv 3$ or $7(\bmod 8)$ when $n=5$ and $\alpha \equiv-1$, $2^{n-3}-1,2^{n-4}-1$ or $2^{n-4}+2^{n-3}-1\left(\bmod 2^{n-2}\right)$ for $n>5$. Again it is easy to check that groups of order $2^{n}$ exist with defining relations $s^{4}=1$ or $s_{n-2}$, $s^{-1} s_{1} s=s_{1}^{\alpha}$ ( $\alpha$ as above). The latter may be written:

$$
\begin{array}{cccc}
s_{1}^{2} s_{2}=1 & s_{n-2} & s_{n-3} & s_{n-3} s_{n-2} \\
{\left[s_{1}, s^{2}\right]=1} & 1 & s_{n-2} & s_{n-2}
\end{array}
$$

where only the first two cases exist for $n=5$. In the last case, by replacing $s$ by $s^{-1}$, we may suppose $s_{1}^{2} s_{2}=s_{n-3}^{-1} s_{n-2}^{-1}=s_{n-3}$; also if $s^{4}=s_{n-2}$ we may suppose (by replacing $s$ by $s s_{1}$ ) that $s^{4}=1$. Thus the last two cases yield one group which is not isomorphic to those defined by the first two cases since it has no abelian subgroup of index 2 . In the second case, if $s^{4}=s_{n-2}$ we may suppose (by replacing $s_{1}$ by $s_{1} s^{2}$ ) that $s_{1}^{2} s_{2}=1$, and this group is not isomorphic to those with $s^{4}=1$ since it has only one normal subgroup of order 2 . Finally, in the case where $s^{4}=1$ and $s_{1}^{2} s_{2} \in \gamma_{n-2}(G)$, if we replace $s_{1}$ by $s_{1}^{\xi}$ we do not affect the value of $s_{1}^{2} s_{2}$, showing that the two groups are not isomorphic. Thus we obtain three groups for $n=5$ and four groups for $n>5$.

(b) Suppose $\gamma_{2}(G)$ has 2 generators and so $s_{2}^{2}, s_{3}^{2} \in \gamma_{4}(G)$. If $s_{3}^{2} \equiv s_{4}\left(\bmod \gamma_{5}(G)\right)$, then the above argument for $s_{1}^{2}$ shows that $s_{2}^{2} \equiv s_{3}\left(\bmod \gamma_{4}(G)\right)$ and so $\gamma_{2}(G)$ is cyclic. Thus we must suppose $s_{2}^{2} \equiv s_{4}\left(\bmod \gamma_{5}(G)\right)$. Proceeding by induction, we obtain

$$
s_{i}^{2} \equiv s_{i+2}(i=2,3, \cdots n-4), s_{n-3}^{2}=s_{n-2}^{2}=1
$$

In particular, if $x \in \gamma_{i}(G)$, then $x^{2} \in \gamma_{i+2}(G)$.

Write $s_{2}^{2}=s_{4} s_{5} x$ and so $s_{3}^{2}=\left[s_{4} s_{5} x, s\right]=s_{5} s_{6}[x, s]$. Since

$$
\begin{aligned}
s^{4} \in Z(G), 1=\left[s_{1}, s^{4}\right] & =\left[s_{1}, s^{2}\right]^{2}\left[s_{1}, s^{2}, s^{2}\right]=s_{2}^{4} s_{3}^{6} s_{4}^{4} s_{5} \\
& =\left(s_{4} s_{5} x\right)^{2} s_{5} s_{6}[x, s] s_{3}^{4} s_{4}^{4} s_{5}=s_{3}^{4} s_{4}^{6} s_{5}^{4} s_{6} x^{2}[x, s] \\
& =\left[s_{2}, s^{4}\right] x^{2}[x, s]=x^{2}[x, s]
\end{aligned}
$$

and so $x \in \gamma_{n-2}(G)$. Proceeding by induction, we get

$$
s_{2}^{2}=s_{4} s_{5} s_{n-2}^{\delta}, \quad s_{i}^{2}=s_{i+2} s_{i+3} \quad(i=3,4, \cdots n-4)
$$

To find $s_{1}^{2}$ and $\left[s_{1}, s_{i}\right]$, we note that

$$
\left[s_{2}, s_{1} s^{2}\right]=\left[s_{2}, s^{2}\right]\left[s_{2}, s_{1}\right]^{s^{2}} \equiv s_{4}\left[s_{2}, s_{1}\right] \quad\left(\bmod \gamma_{5}(G)\right)
$$

and so (by replacing $s_{1}$ by $s_{1} s^{2}$ if necessary) we may suppose that $\left[s_{2}, s_{1}\right] \equiv s_{4}$ $\left(\bmod \gamma_{5}(G)\right)$ and similarly $\left[s_{i}, s_{1}\right] \equiv s_{i+2}\left(\bmod \gamma_{i+3}(G)\right)$ for $i \geqq 2$. Thus, if $s_{1}^{2} \equiv s_{r}\left(\bmod \gamma_{r+1}(G)\right)$, then 


$$
s_{r+2} \equiv\left[s_{r}, s_{1}\right] \equiv\left[s_{1}^{2}, s_{1}\right] \equiv 1\left(\bmod \gamma_{r+3}(G)\right)
$$

and so $r \geqq n-3$. Noting that $s^{4} \in \gamma_{n-2}(G)$, we get

$$
s^{4}=s_{n-2}^{\alpha}, s_{1}^{2}=s_{n-3}^{\beta} s_{n-2}^{\gamma}
$$

Thus $\left[s_{2}, s_{1}\right] s_{2}^{2}=\left[s_{1}^{2}, s\right]=s_{n-2}^{\beta}$ and again, by induction, we get

$$
\left[s_{1}, s_{2}\right]=s_{4} s_{5} s_{n-2}^{\beta+\delta},\left[s_{1}, s_{i}\right]=s_{i+2} s_{i+3} \quad \text { for } i=3,4, \cdots n-4
$$

The equations (1) to (4), together with the fact that $\gamma_{2}(G)$ is abelian, are the defining relations for $G$ and it is easy to check that (for $n \geqq 5$ ) a group of order $2^{n}$ with the given defining relations does exist.

When $n=5, s_{2}^{2}=\left[s_{1}, s_{2}\right]=1$ and so $\beta=\delta=0$. If $s^{4}=1$, then $\left(s_{1} s^{2}\right)^{2}=$ $s_{1}^{2} s^{4} s_{2}^{2} s_{3}=s_{1}^{2} s_{3}$ and so we may suppose $s_{1}^{2}=1$, yielding the 3 stem groups of $\Gamma_{7}$ (see Hall and Senior (1964)).

When $n=6, s_{2}^{2}=s_{4}$ and so $\delta=0$. Replacing $s_{1}$ by $s_{1} s^{2 \beta}$, we may suppose $s_{1}^{2}=s_{4}^{\gamma}$ and $\left[s_{1}, s_{2}\right]=1$, yielding the 4 stem groups of $\Gamma_{23}$ in Hall and Senior (1964).

When $n \geqq 7$, replace $s_{1}$ by $s_{1} s_{n-5}^{\delta}$ and $s_{2}$ by $s_{2} s_{n-4}^{\delta}$. Since $\left(s_{2} s_{n-4}^{\delta}\right)^{2}=s_{2}^{2} s_{n-2}^{\delta}$, we may suppose $\delta=0$. To find the isomorphism classes for these groups, we replace $s$ by $s^{*}=s^{1+2 \xi_{5}} s_{1}^{\zeta}$ and $s_{1}$ by $s_{1}^{*}=s_{1} s^{2 \eta} v$ (as in Theorem 5.2) where $u, v \in \gamma_{2}(G)$. Thus

$$
\begin{array}{r}
s_{2}^{*}=\left[s_{1} s^{2 \eta} v, s^{1+2 \xi} s_{1}^{\zeta} u\right] \equiv s_{2}\left(\bmod \gamma_{3}(G)\right) . \\
{\left[s_{1}^{*}, s_{2}^{*}\right] \equiv\left[s_{1} s^{2 \eta}, s_{2}\right] \equiv\left[s_{1}, s_{2}\right] s_{4}^{\eta} \equiv s_{4}^{1+\eta}\left(\bmod \gamma_{5}(G)\right)}
\end{array}
$$

As we have supposed that $\left[s_{1}, s_{2}\right] \notin \gamma_{5}(G)$, we must take $\eta=0$. Also

$$
\begin{aligned}
\left(s^{1+2 \xi} s_{1}^{\zeta}\right)^{2} & =s^{2+4 \xi} s_{1}^{2 \zeta}\left[s_{1}, s^{1+2 \xi}\right]^{\zeta} \equiv s^{2}\left[s_{1}, s^{2 \xi}\right]^{\zeta}\left[s_{1}, s\right]^{\zeta}\left[s_{1}, s, s^{2 \xi}\right]^{\zeta} \\
& \equiv s^{2}\left(s_{2}^{2} s_{3}\right)^{\xi \xi} s_{2}^{\xi}\left(s_{3}^{2} s_{4}\right)^{\xi \zeta} \equiv s^{2} s_{2}^{\zeta} s_{3}^{\xi \zeta}\left(\bmod \gamma_{n-2}(G)\right)
\end{aligned}
$$

By Corollary 2 of Theorem 3.3, $s^{*}$ is conjugate to $s^{1+2 \xi_{5} \zeta}$ and so

$$
\begin{aligned}
s^{* 4} & =\left(s^{1+2 \xi} s_{1}^{\zeta}\right)^{4}=\left(s^{2} s_{2}^{\zeta} s_{3}^{\xi \zeta}\right)^{2} \\
& =s^{4}\left(s_{2} s_{3}^{\xi}\right)^{2 \zeta}\left(s_{3} s_{4}^{\xi}\right)^{2 \zeta}\left(s_{4} s_{5}^{\zeta}\right)^{\zeta}=s^{4}
\end{aligned}
$$

Similarly, since $\mathscr{C}\left(s_{1}\right)=\left\langle s_{1}, \gamma_{n-3}(G)\right\rangle$ has order 8 , the conjugacy class of $s_{1}$ is $s_{1} \gamma_{2}(G)$ and so $s_{1}^{* 2}=\left(s_{1} v\right)^{2}=\left(s_{1}^{x}\right)^{2}=\left(s_{1}^{2}\right)^{x}$ for some $x \in G$. If $s_{1}^{2} \in \gamma_{n-2}(G)$, then clearly $s_{1}^{* 2}=s_{1}^{2}$ yielding 4 groups. If $s_{1}^{2}=s_{n-3} s_{n-2}^{\gamma}$, take $x=s^{\gamma}$ and so we may suppose $s_{1}^{2}=s_{n-3}$, yielding 2 groups.

THEOREM 5.4. The number of groups $G$ of order $2^{n}$ and class $n-2$ with $G / \gamma_{2}(G)$ of exponent 4 and $\gamma_{1}(G) / \gamma_{2}(G)$ elementary abelian is 6 when $n=5$, 10 when $n=6,14$ when $n=7,16$ when $n>7$ and $n$ odd, and 20 when $n>7$ and $n$ is even. 
Proof. Once again, we use the notation of paragraph 3. By theorem 3.4. $\gamma_{i}(G)=\left\langle s_{i}, \gamma_{1+1}(G)\right\rangle$ for $i=2,3, \cdots n-2$ and $s^{4} \in \mathscr{C}(s) \cap \gamma_{2}(G)=\gamma_{n-2}(G) \leqq Z(G)$. By theorem 3.1, $\gamma_{2}(G)$ may be generated by 3 elements and, since the groups $G$ where $\gamma_{2}(G)$ has 1 or 2 generators were considered in theorem 5.3, we will assume here that $\Phi\left(\gamma_{2}(G)\right)=\gamma_{5}(G)$.

As in the reasoning for Theorem 3.4, we can show that $s_{i} \equiv s_{i-2}^{2}$ $\left(\bmod \gamma_{i+1}(G)\right)$ for $i \geq 5$ and so $G_{1}=\left\langle s, \gamma_{2}(G)\right\rangle$ is a group of order $2^{n-1}$ which satisfies the conditions of theorem 5.3 with $\gamma_{i}\left(G_{1}\right)=\gamma_{i+1}(G)$ for $i=1,2, \cdots n-3$. By noting that, since $s_{2}^{2} \in \gamma_{5}(G), 1 \equiv\left[s_{2}^{2}, s\right] \equiv s_{3}^{2}\left[s_{3}, s_{2}\right] \equiv s_{5}\left[s_{3}, s_{2}\right]\left(\bmod \gamma_{6}(G)\right)$ and so $\left[s_{3}, s_{2}\right] \equiv s_{5}\left(\bmod \gamma_{5}\left(G_{1}\right)\right)$, we may use that theorem to obtain

$$
\left\{\begin{array}{l}
s^{4}=s_{n-2}^{\chi}, s_{2}^{2}=s_{n-3}^{\beta} s_{n-2}^{\gamma},\left[s_{2}, s_{3}\right]=s_{5} s_{6} s_{n-2}^{\beta} \\
{\left[s_{2}, s_{i}\right]=s_{i}^{2}=s_{i+2} s_{i+3}(i>3), s_{3}^{2}=s_{5} s_{6}}
\end{array}\right.
$$

Also (as in theorem 3.4) we may show that

$$
\left[s_{2 j}, s_{1}\right] \equiv s_{2 j+1}\left(\bmod \gamma_{2 j+2}(G)\right),\left[s_{2 j-1}, s_{1}\right] \in \gamma_{2 j+1}(G) \text { for } j \geqq 2 \text {. }
$$

Thus $s_{2 j} \notin \mathscr{C}\left(s_{1}\right)$ and so $s_{1}^{2} \equiv s_{2 r+1}\left(\bmod \gamma_{2 r+2}(G)\right)$ for some $r$. However, since and

$$
\left(s^{2} s_{1}\right)^{2}=s^{4} s_{1}^{2}\left[s_{1}, s^{2}\right] \equiv s_{1}^{2} s_{3}\left(\bmod \gamma_{4}(G)\right)
$$

$$
\left(s_{1} s_{2 r}\right)^{2} \equiv s_{1}^{2} s_{2 r}^{2}\left[s_{2 r}, s_{1}\right] \equiv s_{1}^{2} s_{2 r+1}\left(\bmod \gamma_{2 r+2}(G)\right) \text { for } r>2 \text {, }
$$

we may suppose $s_{1}^{2} \in \gamma_{n-2}(G)$, and so

$$
s_{1}^{2}=s_{n-2}^{\delta} \text { (where } \delta=0 \text { if } n \text { is odd) }
$$

To find $\left[s_{i}, s_{1}\right]$, we note that $s_{1}{ }^{2} \in Z(G)$ and so

$$
\begin{aligned}
1=\left[s_{1}^{2}, s\right] & =s_{2}^{2}\left[s_{2}, s_{1}\right]\left[s_{2}, s_{1}, s_{2}\right] \\
& \equiv\left[s_{2}, s_{1}\right]\left[s_{2}, s_{1}, s_{2}\right]\left(\bmod \gamma_{n-3}(G)\right)
\end{aligned}
$$

Thus $\left[s_{2}, s_{1}\right] \in \gamma_{n-3}(G)$ and so $\left[s_{2}, s_{1}, s_{2}\right]=1$, yielding

$$
\left[s_{2}, s_{1}\right]=s_{2}^{2}=s_{n-3}^{\beta} s_{n-2}^{\gamma}
$$

By theorem $2.3\left[s_{3}, s_{1}\right]=\left[s_{1}, s_{2}\right]^{s_{3}} s_{2}\left[s_{2}, s_{2} s_{3}\right]\left[s_{2}, s_{1}\right]^{s_{2}} s^{-1}$

$$
\begin{aligned}
& =s_{n-3}^{\beta} s_{n-2}^{\gamma} s_{2} s_{5} s_{6} s_{n-3}^{\beta} s_{n-2}^{\gamma} s_{2}^{-1} \\
& =s_{2}\left(s_{5} s_{6}\right) s_{2}^{-1}=s_{5} s_{6}\left[s_{5} s_{6}, s_{2}^{-1}\right] \\
& =s_{5} s_{6}\left[s_{5} s_{6}, s_{2}^{-2} s_{2}\right]=s_{5} s_{6}\left(s_{5} s_{6}\right)^{-2}=s_{5}^{-1} s_{6}^{-1}
\end{aligned}
$$

Similarly, by repeated application of theorem 2.3 , we obtain

$$
\left\{\begin{array}{l}
{\left[s_{4 j-1}, s_{1}\right]=s_{4 j+1}^{-1} s_{4 j+2}^{-1},\left[s_{4 j}, s_{1}\right]=s_{4 j+1} s_{4 j+2}} \\
{\left[s_{4 j+1}, s_{1}\right]=1,\left[s_{4 j+2}, s_{1}\right]=s_{4 j+3} \text { for } j \geqq 1}
\end{array}\right.
$$


Once again the equations (1) to (4), together with the fact that $\gamma_{3}(G)$ is Abelian, may be shown to define a group of order $2^{n}$ when $n>5$.

When $n=6$, we have $s_{s}^{2}=s_{3}^{2}=s_{4}^{2}=1,\left[s_{i}, s_{j}\right]=1$ for $i, j=1,2,3,4$, $s^{4}=s_{4}^{\alpha}$ and $s_{1}^{2}=s_{4}^{\delta}$. Since $\left(s s_{1}\right)^{4}=s^{4} s_{2}^{2}\left[s_{2}, s^{2}\right]=s^{4} s_{4}$ we may suppose (by replacing $s$ by $s s_{1}$ ) that $\alpha=0$ giving the 2 stem groups of $\Gamma_{22}$ in Hall and Senior(1964).

When $n=7$, we must have $s_{2}^{2} \in \gamma_{5}(G)$, and so

$$
\begin{gathered}
s^{4}=s_{5}^{\alpha}, s_{1}^{2}=s_{4}^{2}=s_{5}^{2}=1, s_{2}^{2}=\left[s_{2}, s_{1}\right]=s_{5}^{\gamma}, \\
s_{3}^{2}=\left[s_{3}, s_{2}\right]=\left[s_{3}, s_{1}\right]=\left[s_{4}, s_{1}\right]=s_{5} .
\end{gathered}
$$

By theorem 5.3, we cannot reduce this any further unless we replace $s$ by $s s_{1} t$ where $t \in\left\langle s^{2}, \gamma_{2}(G)\right\rangle=K$. However $\left(s s_{1} t\right)^{2}=s^{2}\left(s_{1} t\right)^{2}\left[s_{1} t, s\right]$

$$
=s^{2} s_{1}^{2} t^{2}\left[t, s_{1}\right] s_{2}\left[s_{2}, t\right][t, s] \equiv s_{2} s^{2}\left(\bmod \gamma_{3}(G)\right)
$$

Since $\gamma_{3}(G)^{2}$ and $\left[\gamma_{3}(G), K\right] \leqq \gamma_{5}(G)$, this yields $\left(s s_{1} t\right)^{4} \equiv s^{4} s_{2}^{2}\left[s_{2}, s^{2}\right] \equiv s_{4}$ $\left(\bmod \gamma_{5}(G)\right)$, contradicting (1) and so we may not replace $s$ by $s s_{1} t$. Thus there are 4 isomorphism classes of groups in this case.

When $n>7$, we may show (as in Theorem 5.3) that $s_{2}^{2}$ may be taken to be $s_{n-3}, s_{n-2}$ or 1 , and (as in the case when $n=7$ ) that different values of $s^{4}$ yield non-isomorphic groups. In the case where $s_{2}^{2}=s_{n-3}$, by replacing $s_{1}$ by $s_{1} \delta_{2}^{\delta} s_{3}^{\delta} s_{4}^{\delta}$, we may replace $s_{1}^{2}$ by $s_{1}^{2} s_{3}^{2 \delta}\left[s_{3}, s_{2}\right]^{\delta}=1$. In the case where $s_{2}^{2} \in \gamma_{n-2}(G)$ and $n$ is even, we have $s_{1}^{2}=s_{n-2}^{\delta}(\delta=0$ or 1$)$. If we replace $s_{1}$ by $s_{1}^{*}=s_{1} w$ with $w \in \gamma_{3}(G)$, then $s_{1}^{* 2}=\left(s_{1} w\right)^{2}=s_{1}^{2} w^{2}\left[w, s_{1}\right]$. Since $\gamma_{3}(G)$ is generated by $s_{3} s_{4}$ and $s_{3}$, we may write $w=\left(s_{3} s_{4}\right)^{\omega} s_{3}^{\tau}$ for some $\omega, \tau$ and so $w^{2}=s_{5}^{-\omega+\tau} s_{6}^{\tau},\left[w, s_{1}\right]=s_{5}^{-\tau} s_{6}{ }^{-\tau}$ and $s_{1}^{* 2}=s_{1}^{2} s_{5}^{-\omega}$. Since $s_{1}^{* 2} \in \gamma_{n-2}(G), \omega=0$ and so $s_{1}^{* 2}=s_{1}^{2}$. If we replace $s_{1}$ by $s_{1}^{*}=s_{1} s_{2} w$, then

$$
\begin{aligned}
s_{1}^{* 2}=\left(s_{1} s_{2}\right)^{2} w^{2}\left[w, s_{1} s_{2}\right] & =s_{1}^{2} s_{2}^{2}\left[s_{2}, s_{1}\right] w^{2}\left[w, s_{2}\right]\left[w, s_{1}\right]\left[w, s_{1}, s_{2}\right] \\
& =s_{1}^{2}\left[w, s_{1}\right]\left[w, s_{1}, s_{2}\right] \text { by (3) and (4) } \\
& =s_{1}^{2}\left[w, s_{1}\right] \text { since }\left[w, s_{1}\right] \in \gamma_{n-2}(G) \\
& =s_{1}^{2} s_{5}^{-\tau} s_{6}^{-\tau}
\end{aligned}
$$

Again $s_{1}^{* 2} \in \gamma_{n-2}(G) \Rightarrow \tau=0 \Rightarrow s_{1}^{* 2}=s_{1}^{2}$ and so the two values for $\delta$ give non-isomorphic groups.

Thus, when $n>7$ and $n$ is odd the six groups are given by $s^{4}=s_{n-2}$ or $1, s_{1}^{2}=1$ and $s_{2}^{2}=s_{n-3}, s_{n-2}$ or 1 ; when $n>7$ and $n$ is even the ten groups are given by $s^{4}=s_{n-2}$ or 1 and

$$
\begin{aligned}
& s_{2}^{2}=s_{n-3} \quad s_{n-2} \quad s_{n-2} \quad 1 \quad 1 \\
& \begin{array}{lllll}
s_{1}^{2}=1 & s_{n-2} & 1 & s_{n-2} & 1
\end{array}
\end{aligned}
$$

The results of theorems 5.1 to 5.4 yield the results of theorem 1.2. 


\section{References}

N. Blackburn (1958), 'On a special class of p-groups', Acta Math. 100, 45-92.

N. Blackburn (1957), 'On prime-power groups in which the derived group has two generators', Proc. Camb. Phil. Soc. 53, 19-27.

M. Hall and J. K. Senior (1964), The Groups of Order $\mathrm{a}^{n}$; ( $\left.n \leqq 6\right)$, (The Macmillan Company, New York, 1964).

P. Hall (1933), 'A Contribution to the theory of groups of prime-power order', Proc. Lond. Matk. Soc. (2) 36, 29-95.

R. James and J. Cannon (1969), 'Computation of isomorphism classes of p-groups', Maths. of Computation 23, 135-140.

The University of New South Wales

Sydney, Australia 